trust for the same time period. From this data we could estimate the proportion of lung cancer patients from each trust that were referred for mediastinal staging with EBUS.

Results In 2012, 2302 patients were diagnosed with lung cancer in this Network. In the same period, 193 patients were referred for EBUS mediastinal staging (8.4\%). The proportion of lung cancer patients referred for mediastinal staging with EBUS varied significantly across the ten trusts ranging from $3.4 \%$ to $30.2 \%$ $(\mathrm{p}<0.0001)$. The spearman co-efficient was $0.60(\mathrm{p}=0.07)$ suggesting a possible relationship between the proportion of patients referred for EBUS and surgical resection (Figure 1). However, this may be due to a very high rate of staging EBUS at one trust, which if excluded yields a spearman co-efficient of $0.45(\mathrm{p}=0.22)$.

Discussion It is highly concerning that only $8 \%$ of lung cancer patients underwent EBUS nodal staging in our network given $52 \%$ of UK patients with histologically confirmed NSCLC are stage I-III at the time of diagnosis. It is of note that the Trust with the highest proportion of patients undergoing EBUS nodal staging have the highest surgical resection rate and three of the four Trusts with the lowest resection rates refer $<5 \%$ of patients for EBUS nodal staging. Standardisation of referral practice across the Network is a key future goal for the EBUS Sub-group.

\section{P216 LOCAL ENDOBRONCHIAL ULTRASOUND (EBUS) SERVICE REDUCES WAITING TIME FOR TEST RESULTS}

JL Dickson, M Lawson. Broomfield Hospital, MEHT, Chelmsford, UK

\subsection{6/thoraxjn-2014-206260.345}

Introduction Endobronchial ultrasound (EBUS) is increasingly used in investigating mediastinal lymphadenopathy. A recent Thorax paper suggested EBUS should only be performed in large centres to ensure quality. A recently established EBUS service at Broomfield Hospital appeared to reduce the time taken to obtain results compared to the regional service previously used. The service was audited to ensure quality and evaluate changes to pathway times.

Objectives Compare time from decision for EBUS to test result between local and regional service and ensure safety and accuracy.

Methods Data were collected prospectively for all EBUS cases after the local service was established in August 2013. Accuracy and safety of the service were audited based on the first 8.5 months of operation. Data were extracted from the MDT database for patients referred to the regional service between November 2013 and January 2014. Time from MDT decision for EBUS referral and subsequent MDT discussion of results were compared between both services. Data were compared using the Mann-Whitney $\mathrm{U}$ test using the statistics package in R. Results Average time from decision to EBUS result was 19 days in the local service based on the first 21 cases performed. There was a 40 day average turnaround for the regional service based on the 10 cases referred between November 2013 and January 2014. This represented a statistically significant reduction in waiting time of 21 days $(\mathrm{p}=0.0001)$. The local service was safe and accurate with no reported complications in 42 cases over the first 8.5 months and an overall accuracy of $88 \%$ increasing to $94 \%(31 / 33)$ in suspected cancer cases.

Conclusions Our recently established local EBUS service is safe and accurate but also significantly reduces the time between decision to EBUS and test results discussed in MDT compared to the regional referral service previously used. This is important for patients on the 62-day cancer pathway. A local service enables people to undergo investigations nearer to home. The establishment of national reference standards for EBUS is important to ensure optimal quality but this can be achieved in local services which may offer additional benefits to patients.

\section{P217 THE NEGATIVE PREDICTIVE VALUE OF ENDOSONOGRAPHY FOR MEDIASTINAL STAGING OF NON-SMALL CELL LUNG CANCER}

${ }^{1} \mathrm{~K}$ Sayal, ${ }^{2} \mathrm{M}$ Scarci, ${ }^{3} \mathrm{~N}$ Carroll, ${ }^{1} \mathrm{~B}$ Dougherty. ${ }^{1}$ Department of Thoracic Oncology, Papworth Hospital NHS Foundation Trust, Papworth Everard, Cambridge, UK; ${ }^{2}$ Department of Thoracic Surgery, Papworth Hospital NHS Foundation Trust, Papworth Everard, Cambridge, UK; ${ }^{3}$ Department of Radiology, Cambridge University NHS Foundation Trust, Cambridge, UK

\subsection{6/thoraxjnl-2014-206260.346}

Introduction and objectives The ASTER trial demonstrated that the sensitivity of combined endobronchial/endoscopic ultrasound [EBUS/EUS] is similar to that of mediastinoscopy. ${ }^{1}$ NICE guidelines now recommend combined EBUS/EUS for initial mediastinal staging as an alternative to surgical staging. ${ }^{2}$ Although surgical staging following negative endosonography is recommended, ASTER showed that 11 mediastinoscopies are required to identify one case of mediastinal disease. We aimed to determine the negative predictive value of EBUS/EUS for mediastinal staging in our practice.

Method We identified all patients who had undergone primary surgical resection with lymph node sampling for NSCLC between May 2012 and April 2014 previously staged with CT, PET-CT, EBUS+/-EUS and/or mediastinoscopy. Demographic,

\begin{tabular}{lll} 
Abstract P217 Table 1 & Patient characteristics \\
\hline & Number & Percentage (\%)(\% \\
\hline Median age & 68 years & \\
Range & $43-87$ years & \\
Histology: & & \\
Squamous & 19 & 45 \\
Adenocarcinoma & 19 & 45 \\
Other & 4 & 10 \\
EBUS only staging & 35 & 83 \\
EUS only staging & 1 & 2 \\
Combined EBUS/EUS staging & 6 & 14 \\
Pre-EBUS/EUS PET/CT staging: & & \\
T1 & 10 & 24 \\
T2 & 23 & 55 \\
T3 & 7 & 17 \\
T4 & 2 & 5 \\
Pre-EBUS/EUS PET/CT staging: & & \\
N0 & 9 & 21 \\
N1 & 13 & 31 \\
N2 & 10 & 24 \\
N3 & 6 & 14 \\
Equivocal & 4 & 10 \\
Tumour localization: & 13 & 26 \\
Left lower lobe & 11 & \\
Left upper lobe & & \\
Right upper lobe & & \\
Right middle lobe & & \\
Right lower lobe & & \\
\hline & 11 & \\
\hline & & \\
\hline
\end{tabular}

